POLLACK PERIODICA

An International Journal for Engineering and Information Sciences

DOI: $10.1556 / 606.2017 .12 .1 .6$

Vol. 12, No. 1, pp. 69-80 (2017)

www.akademiai.com

\title{
METHODOLOGY FOR CLINICAL INTEGRATION OF E-HEALTH SENSOR-BASED SMART DEVICE TECHNOLOGY WITH CLOUD ARCHITECTURE
}

\author{
${ }^{1}$ Ábel GARAI, ${ }^{2}$ István PÉNTEK, ${ }^{3}$ Attila ADAMKÓ, ${ }^{4}$ Ágnes NÉMETH \\ 1,2,3 Department of Information Technology, Faculty of Informatics, University of Debrecen \\ Kassai út 26, H-4028 Debrecen, Hungary, e-mail: ${ }^{1}$ garai.abel@inf.unideb.hu \\ 2pentek.istvan@inf.unideb.hu ${ }^{3}$ adamko.attila@inf.unideb.hu \\ ${ }^{4}$ Department of Pulmonology, $2^{\text {nd }}$ Pediatrics Clinic, Semmelweis University \\ Tűzoltó u. 7-9, H-1094 Budapest, Hungary, e-mail: nemeth.agnes1@med.semmelweis-univ.hu
}

Received 24 August 2016; accepted 9 December 2016

\begin{abstract}
The international healthcare systems interoperability is an unresolved technological area at the moment. This paper demonstrates the results of the software engineering research for simplex syntactic and semantic technical interoperability of hospital information systems, eHealth smart device technology and clinical telemedicine instruments through the recently developed Telemedicine Interoperability Hub. Several similar experiments exist. This research is unique in building a prototype interconnecting not only healthcare information systems with each other, but aiming to establish a general healthcare interoperability scheme including also the eHealth smart devices and telemedicine instruments. The aim of this research is to establish cloud-based data interchange capability with the newly developed information technology system interconnected with the emerging eHealth Internet of Things solutions and the classical hospital information system architecture. Notwithstanding the international information technology medical data exchange standards, like Health Level Seven, the adoption of an industry-wide open telemedicine syntactic and semantic interoperability standard is necessary. The research studied varying simplex, duplex, full-duplex, data package- and file-based information technology modalities establishing stable system interconnection among clinical instruments, healthcare systems and eHealth smart devices. This research is the manifestation of the trilateral cooperation of the University of Debrecen Department of Information Technology, Semmelweis University Second Paediatric Clinic and T-Systems Healthcare Competence Center Central and Eastern Europe. The developed experimental software engineering solution was embedded in hybrid cloud architecture after testing private cloud Infrastructure-as-a-Service and Public Cloud Software-as-a-Service technical solutions.
\end{abstract}

Keywords: Hybrid cloud architecture, Cloud Architecture, Hospital information system, Internet of things, Health Level Seven, Smart devices, Telemedicine, Spirometry 


\section{Introduction}

The evolution of healthcare information technology determines the overall delivered healthcare service level. International Healthcare Information Systems (HIS) interoperability relies upon global communication technology backbone networks. Notwithstanding the empirically possible global healthcare interoperability, technological, legal, financial and social considerations block the wide-spreading proliferation of feasible overwhelming solutions.

The general international healthcare systems interoperation is still only a vision. This research program intends to make a significant step towards this general interoperability. There are several similar initiatives all around the world. However, these focus only on one single domain, like hospital information systems, telemedicine equipment or eHealth devices. This program has a unique approach, as it intends to establish a general healthcare interoperability scheme. This interoperability scheme includes the hospital information system domain, the telemedicine domain and the eHealth smart device domain. This research program brings all these different and separated domains closer to each other. The research aims to establish an executable interoperability prototype interconnecting all these three different domains.

Standardization initiatives, like Health Level Seven (HL7, [1]) provide international interoperability capability for regional HIS [2]. The information and communication systems on the western hemisphere supply with the necessary data transmission quality of service for international healthcare information exchange. This quality is critical for the healthcare data transmission. For example, data package-based interoperability requires continuous online network availability. High network availability is required for reliable healthcare data exchange. Beside the classical HIS landscape telemedicine appliances and body-sensor enabled smart devices penetrate the healthcare supply chain [3]. The Open Telemedicine Interoperability Hub (OTI-Hub, Fig. 1) presented in this paper provides interoperability platform for international healthcare information interchange bridging the interoperability gap among classical HIS, telemedicine instruments and eHealth smart-devices.

Free movement of persons and epidemic control imply the necessity of flawless international healthcare systems interoperability [4], [5], [6]. The universal proliferation of meaningful multinational healthcare data exchange requires further significant efforts at conceptual, technical, organizational and at regulatory level [7]. For this purpose, this research establishes value at conceptual and technical level in this field of science.

\section{Clinical practice and eHealth technology embedded in hybrid cloud}

Cloud architectures are classified into private, public and hybrid clouds [8]. The private cloud offers feasible technical solution for the sensitive personal patient data, and the public cloud delivers the necessary scalability. Therefore, the final technical architecture falls under the hybrid architecture category. The applied cloud-based HIS, eMedsol, operates in sixty hospitals and serves forty thousand users in Hungary, Romania, Czech-Republic and Bosnia-Herzegovina. The eMedSol is equipped with Unix-based (Linux, AIX HP-UX, SCO) WebSphere Application Server (V5, V6) 
interconnected with Oracle (10gR2) and Progress (V10 - OpenEdge) relational database management systems (RDBMS). Each installation (for clinical institution category under three hundred beds) is equipped with a primary and secondary virtual server, two quad-CPUs and 16 GB Memory. The OTI-Hub is embedded in Google public cloud (Google Cloud Platform), and the sensitive patient personal data is stored on the Open Telekom Cloud, so these data remains within the EU according to the corresponding München-based datacenter. The Cloud-based healthcare data can be later analyzed with Big Data [8] analytics.

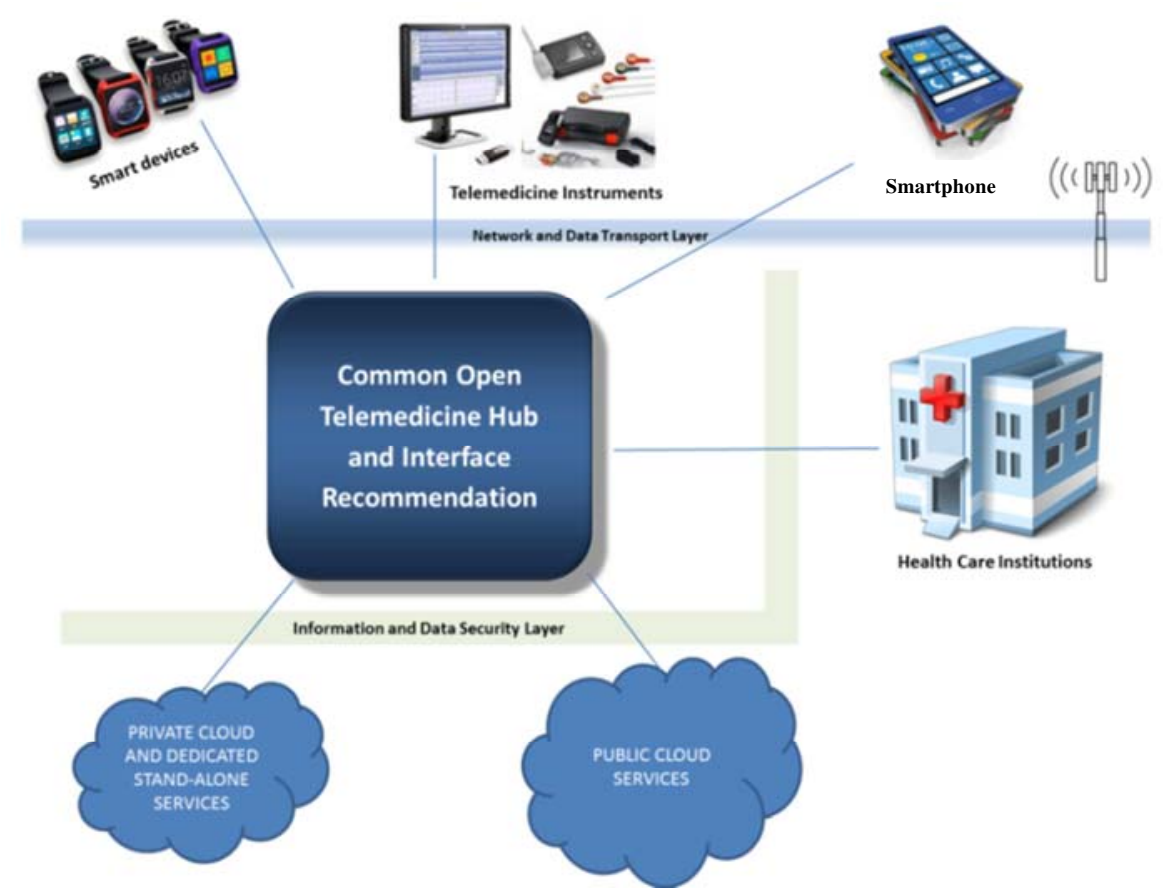

Fig. 1. Open Telemedicine Interoperability Hub (OTI-Hub)

Building a hybrid cloud is a significant information engineering challenge. Hybrid cloud means that at least two or more architecturally different cloud environments need to operate together. The network connection between the private and public cloud datacenters, the firewall settings, the deployed protocols and many other factors should be synchronized for building hybrid cloud architecture. Therefore, when a research program, like this, decides to establish hybrid cloud architecture, it requires resolving a significant bunch of technical burdens.

Insulin pumps developed for diabetes with sensors implanted under the skin require stable data-circulation. This solution supplemented by cloud-based interface and dataprocessing programs transfer the actual blood sugar level to the designated diabetes center through telecare and telemedicine software systems. The actual patient blood sugar information is displayed directly at the diabetes center, and the blood sugar 
timelines, periods are monitored customized according to the emerging patient complaints. The other significant group of endemic is patients suffering from asthma. A mobile telemedicine device (Fig. 2) has been developed for asthmatic patients that should be blown by the patient at home and the results are automatically uploaded to the asthma clinical center through the Internet. The specialist analyzes the results during the subsequent personal patient visit or conducts a remote determination of the necessary medical intervention. The medical professional evaluates the spirometry test results based on criteria system standard. Similar principles apply to the heart patients' ECG tests and to the blood pressure monitoring.

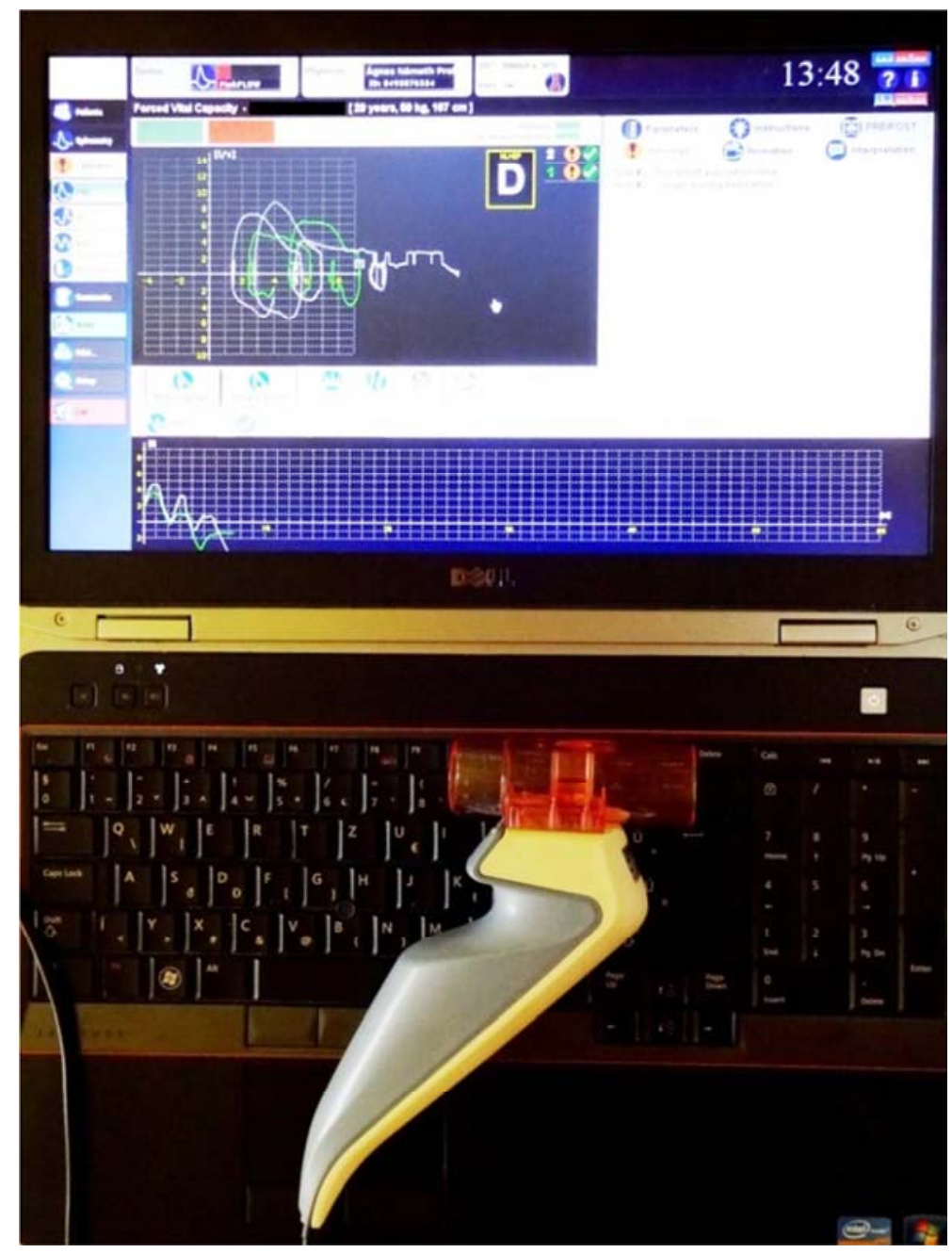

Fig. 2. Data-exchange over OTI-Hub with spirometer PDD-301/shm 


\section{Research background}

HL7 healthcare interoperability de facto international standard [9] and the widely recognized Systematized Nomenclature of human Medicine Clinical Terminology (SNOMED-CT, [10]) nomenclature have been appointed for this research as prevailing conditions. The conceptual logic of the developed OTI-Hub (Fig. 3) relies upon the basis of the aforementioned standard and nomenclature. Theoretically the vast majority of the contemporary HIS and medical instruments are HL7-enabled [11]. However, in reality the HL7 standard itself has different, not fully compatible sub-versions (HL7 v2.x, HL7 v3) [12]. The presumption that the latest HL7 v3 version is the generally most applicable, based on the experience of the study is not satisfied. The reality is that healthcare institutions, that already introduced the most widespread HL7 v2.x-based Information technology (IT) landscape (e.g. University of Debrecen, Clinical Department or Semmelweis University), stick to that version and a potential upgrade to the latest HL7 v3 standard is absolutely not foreseen by these institutions in the near future [12]. Therefore, the Research Team decided in favor of the HL7 v2.3.1 standard during the research because of its acceptance by all parties involved.

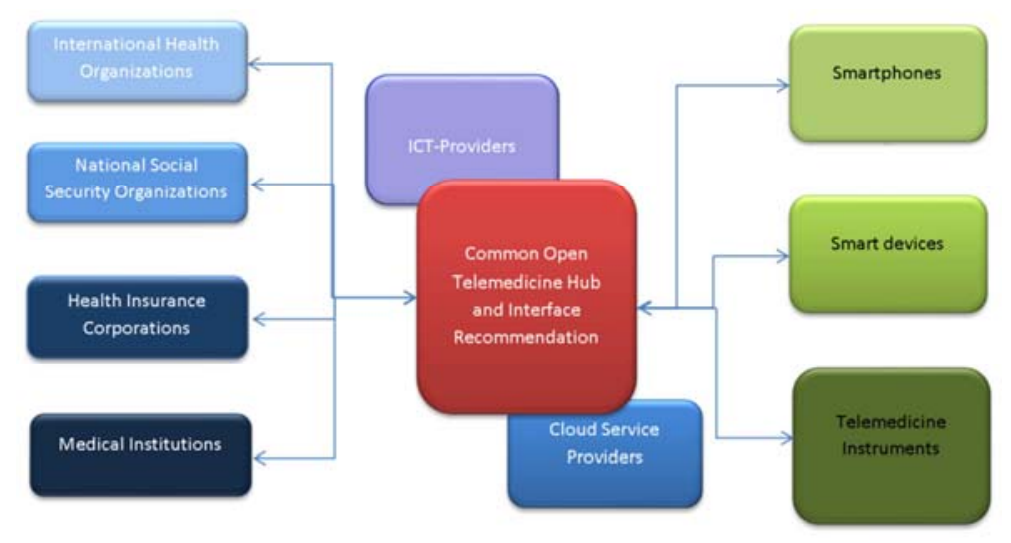

Fig. 3. Logical structure of Open Telemedicine Interoperability Hub (OTI-Hub)

\section{Methodology}

Clinical systems interoperability reaches beyond plain data-exchange: it constitutes interoperability at technical, semantic and at process level. In the empirical model of the research the OSI model (ISO/IEC 7498-1, [13] is mapped against the aforementioned interoperability levels, therefore these three interoperability modalities are interpreted also at the corresponding information technology abstraction layer within this article. Technical and semantic interoperability is targeted within the presented research. Among the technical interoperability modalities instead of the TCP/IP the file-based interface connection has been elected, as this option offered significantly more flexibility during the research [14]. The clinical spirometer PDD-301/shm has been 
linked over USB connection to the corresponding spirometry desktop program provided by the manufacturer (Fig. 4).

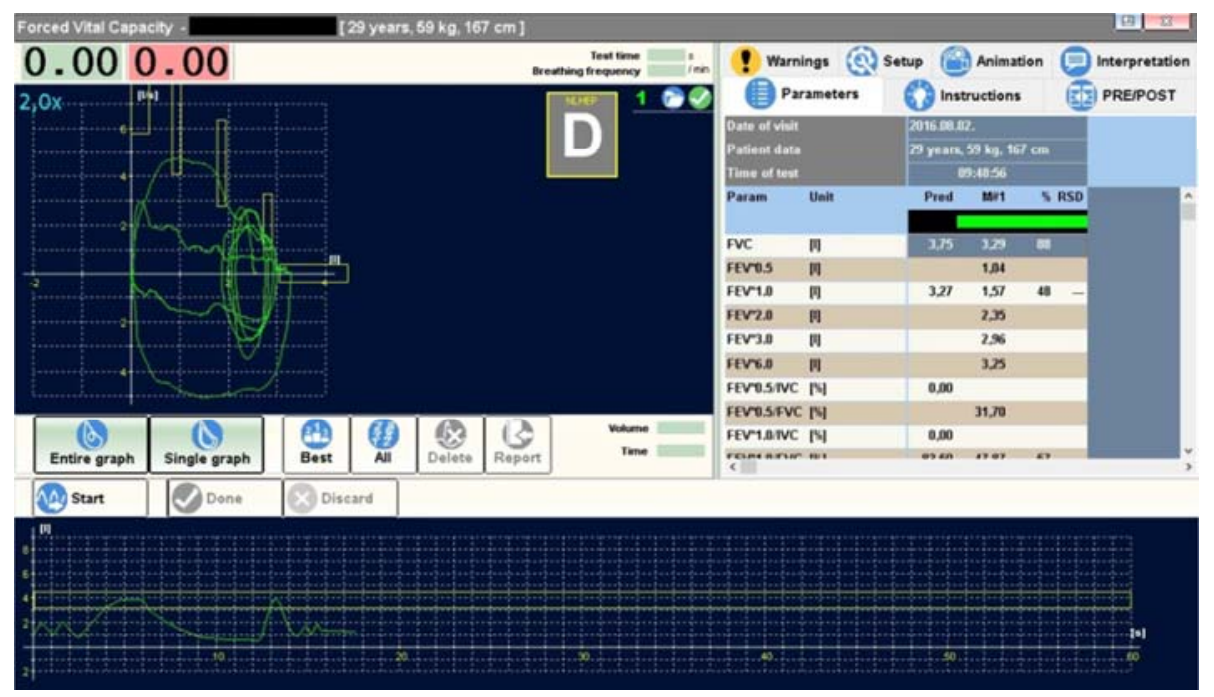

Fig. 4. Forced Vital Capacity (FVC) spirometry test result with PDD-301/shm spirometer

The spirometry desktop program has been installed on a standalone Dell Latitude E6520 laptop equipped with Windows 10 operating system. The spirometer has been calibrated by the manufacturer for the research. Forced vital capacity spirometry test has been undertaken with a healthy individual. Having the test results stored in the spirometry desktop software (Fig. 4), the HL7 v2.3.1 interface file has been exported. This interface file has been processed by the cloud-based OTI-Hub. The OTI-Hub appended the spirometry information with the previously transformed cardio bodysensor information captured by the L18 Smart Bluetooth Wristband. The generated HL7 interface file is imported after parameterization into the factory acceptance test instance of the MedSol HIS. Both the imported spirometry and cardio test results are retrieved and displayed by the patient report query of the hospital information system. The information technology result is validated by the Department of Information Technology, University of Debrecen and by T-Systems Healthcare Competence Center. The clinical result is validated by the Semmelweis University $2^{\text {nd }}$ Department of Pediatrics.

\section{Technical system integration through open telemedicine interoperability hub}

Interoperability is two or more systems' capability for data exchange. Syntactic interoperability refers to the technical ability of systems data interchange. Syntactic interoperability means, that the data package or file emitted by the sending system is 
technically readable by the recipient system. For example, file coding and character coding play a significant role in syntactic interoperability. Semantic interoperability refers to the information exchange modality, when the interchanged information is meaningful for the recipient system. For example, it is technically possible, that the emitting system sends technically interpretable numbers to the recipient system, and the recipient system stores there received numbers in the name field. The syntactic interoperability was fulfilled, as the data exchange was successful. However, the semantic interoperability failed, because a readable name was logically expected, however pure numbers were sent and stored under the name field in this case.

The goal was to create a flexible telemedicine interoperability hub system to extend the classical hospital systems [15]. To achieve this goal, industry-wide accepted technologies have been applied. Also, the software development environment for the hub (Fig. 5) has been selected. All the selected technologies are available also on Microsoft stack. The Hub has been implemented with Microsoft stack and further open source technologies [16].

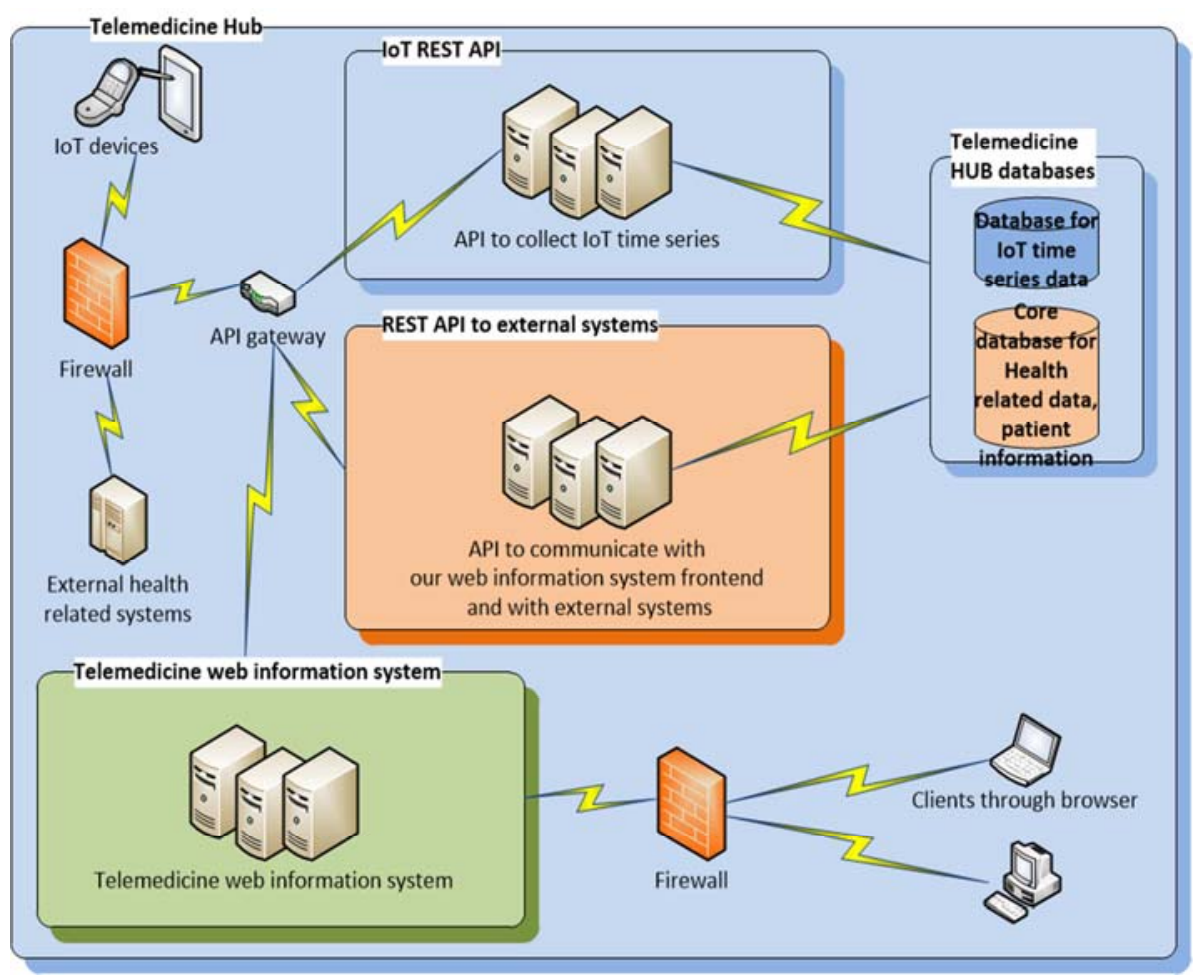

Fig. 5. Internal Design for Open Telemedicine Interoperability Hub 
The following technologies are applied within the OTI-Hub and in its modules:

- Receiver module handles the received measurement data through http web request. This module is implemented via Microsoft Web API, and the message format is JSON and XML. This module uses Open Authentication (OAuth) protocol to authorize and authenticate the users and devices. The REST principles have been adapted as far as possible [17];

- Transformation module operates with the collected data by the receiver module and the main task of this module is data transformation to HL7 format. This module was implemented in $\mathrm{C \#}$ as a Windows service [18], [19];

- Data storage module is responsible for building data warehouse from the collected and transformed data. This module was implemented in Apache Hadoop software library;

- Interpreter module works with the data warehouse module and its main task is to interpret the collected and aggregated data. This module works with Apache Hadoop software library, too [20];

- The most important module is the Hub integration module. This module offers export data feature from the telemedicine hub into external systems, e.g.: hospital information systems. This module uses REST API endpoints to transport data into external systems; and it applies the technologies summarized at the receiver module (Microsoft Web API, OAuth, JSON and XML);

- Real-time communication module operates with SignalR technology and its main task is maintaining an open socket between the Hub and the devices. This module is critical when the device is recording data frequently and the received data must be available prompt on the Hub. In this case, when lower priority measurement data is not necessarily need to be available on the Hub immediately, then this module can be excluded from the processing chain [21], [22]. The socket gives the ability to use a channel between the Hub and the device without reconnection and re-authentication. The only significant latency is the network latency in this case [23]. SignalR software library is available in the most popular development environment [24].

\section{Results}

The over OTI-Hub transferred spirometry and cardio sensory HL7 test result data has been successfully imported, interpreted and presented in the target HIS. As expected, the correct spirometry and cardio test results reflected back through the HIS's patient result query. The cardio information in the HIS query shows the values 
transformed by OTI-Hub. The results show that HL7-based health data interchange among different IT architectures is completely feasible. The OTI-Hub successfully provides seamless interoperability among Android-, Unix- and Windows-platforms. Both cloud and standalone architecture components were effectively interconnected during the research. The HL7-based information exchange among the healthcare information acceptance system, the dedicated standalone spirometry system and the OTI-Hub was stable, trackable and successful. The combination and reconciliation of the different HL7 versions are handled successfully within the OTI-Hub logic. The conversion of the body-sensory smart device output data stream into meaningful HL7 interface information is a significant challenge [25]. The spirometry output interface file was emitted and processed by the OTI-Hub correctly:

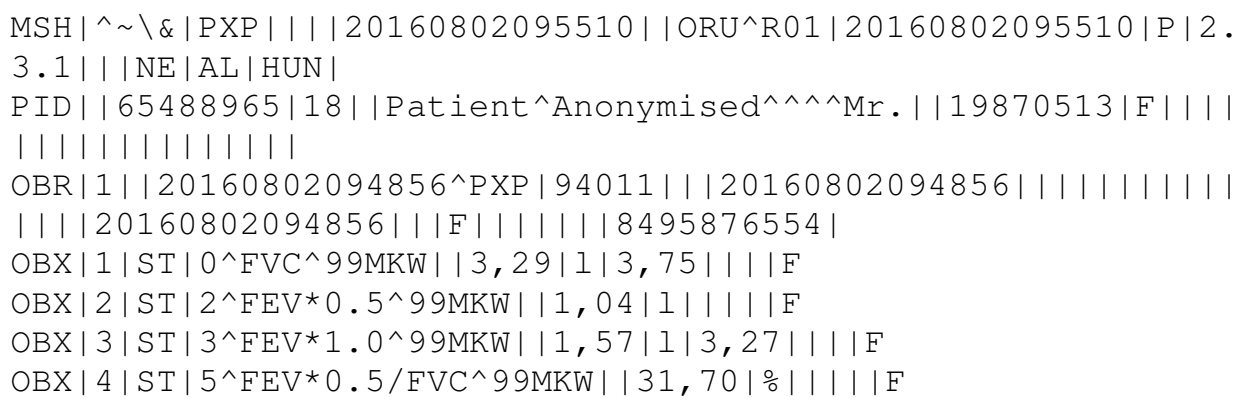

The healthcare smart-device manufacturers generally provide unspecified output format [26]. These smart device output formats are mostly readable for humans, but not exact enough for electronic processing [27], [28]. Also, the healthcare smart device output data streams are not ready to be processed by HIS unless they are transformed into meaningful static values. Although the OTI-Hub successfully interpolated the healthcare smart device data stream into interpretable HL7 values, this area calls for further examination. As healthcare smart devices originating from different manufacturers offer diverse output data-format and data emission frequency [29], there is room for overall standardization in this field. The research did not analyze the backward data-flow, e.g. when data originating from classical HIS sends data to healthcare smart app running on smartphone through the OTI-Hub. This backward healthcare information flow is to be examined in the course of a separate research. The research results satisfied the requirements, however provided significant lessons learned, illustrated in the conclusion.

\section{Conclusion}

The aim of the research was to set up a hybrid cloud-based prototype appending the clinical research patient information with the smart-device body-sensory information flow. The original scientific question was, if the body-sensory smart device technology and telemedicine instrument architecture can be integrated within the classical healthcare information landscape. Based on the results and experiences of the demonstrated research, it is possible namely the flawless interconnection of the body- 
sensory Internet of Things (IoT) smart device technology, the telemedicine architecture and the classical healthcare information technology landscape. The research illustrates that meaningful healthcare information exchange is possible between HL7 capable HIS and eHealth smart devices. This experiment demonstrated one possible healthcare interoperability solution. It proved that interoperability among the hospital information systems, eHealth smart devices and telemedicine domain is possible. The next phase of the research will contain the bidirectional or multidirectional interoperability among these three different domains. Another important finding of the experiment is that the interconnection of different platforms (Unix, Windows) requires special nesting software classes due to the party different line feed and carriage return coding (LF on Unix, LF+CR on Windows). The non-English character coding between Unix and Windows also needs attention. The drafted Open Telemedicine Interoperability Hub serves with the methodological basis and software solution for the interconnection of the world of IoT and classical healthcare technology. Through the healthcare smart device technology unprecedentedly much human body-sensory data will be generated and made available in the foreseeable future. This bulk information base serves inter alia as important input for epidemic control and set new targets for pharmaceutical development. Big Data analytics methodologies foster pattern and trend analysis based on the captured body-sensory healthcare information base. The hybrid cloud architecture assures the essential scalability for the OTI-Hub in order to bear with the necessarily robust transaction processing capacity. The illustrated architectural topology and systems integration solution provides technological solution for the integration of international body-sensory, telemedicine and classical healthcare data exchange. Notwithstanding that the illustrated research results offer some optimism, however the ruling national healthcare data-related legal prerequisites need to be internationally harmonized for enabling the required break-through. The illustrated OTI-Hub solution provides international eHealth data-exchange. Furthermore, the demonstrated cloudbased eHealth interoperability solution provides framework for additional application areas, like cloud-based implementation of the da Vinci Surgical System.

\section{Acknowledgements}

The Authors thank Gábor Aszódi, Kálmán Fábián for the organizational and technical support, and László Csatár for providing the PDD-301/shm spirometer for the research.

\section{References}

[1] Health Level Seven Standard Version 2.3.1, An application protocol for electronic data exchange in healthcare environments, Health Level Seven International, http://www.hl7.org/ (last visited 6 April 2016).

[2] Fong B., Fong A. C. M., Li C. K. Telemedicine technologies: Information technologies in medicine and telehealth, Chichester, Wiley, 2011. 
[3] Asada H. H., Shaltis P., Reisner A., Rhee S., Huthinson R. C. Mobile monitoring with wearable photoplethysmographic biosensors, IEEE Engineering in Medicine and Biology Magazine, Vol. 22, No. 3, 2003, pp. 28-40.

[4] Eren H., Webster J. G. The e-medicine, e-health, m-health, in Telemedicine and Telehealth Handbook, Oakville, CRC Press, 2015.

[5] Varshney U. Pervasive healthcare and wireless health monitoring, Mobile Networks and Applications, Vol. 12, No. 2, 2007, pp. 113-127.

[6] Rossi R. J. Applied biostatistics for the health sciences, Hoboken, NY, Wiley, 2010.

[7] Martinez L., Gomez C. Telemedicine in the 21st Century, Applied biostatistics for the health sciences, Nova Science Publishers, NY, 2008.

[8] Definition of Big Data, Merriam-Webster's Collegiate Dictionary, http://www.merriamwebster.com/dictionary/big\%20data, (last visited 4 July 2016).

[9] Chandrasekaran S., Mohan S., Natarajan R. Survey on health cloud characteristics, Health and Technology, Vol. 5, No. 2, 2015, pp. 135-146.

[10] International Health Terminology Standards Development Organization (IHTSDO), Copenhagen, http://www.ihtsdo.org (last visited 10 June 2016).

[11] Bashshur R. L., Shannon G. W. History of telemedicine: evolution, context, and transtiormation, New Rochelle, NY, Mary Ann Liebert, 2009.

[12] Xiao Y., Tao Y., Li Q. A new wireless Web access mode based on cloud computing, Pacific-Asia Workshop on Computational Intelligence and Industrial Application, PACIIA '08, Wuhan, China, 19-20 December 2008, Vol. 1, 2008, pp. 645-649.

[13] ISO/IEC 7498-1:1994 Information technology, Open systems interconnection, Basic reference model: The basic model (OSI-Model), International Organization for Standardization (ISO), http://www.iso.org/iso/catalogue_detail.htm?csnumber=20269, (last visited 6 June 2016).

[14] Baranyai P., Csapó Á. Definition and synergies of cognitive infocommunications, Acta Polytechnica Hungarica, Vol. 9, No. 1, 2012, pp. 67-83.

[15] Adamkó A., Garai Á., Péntek I. Common open telemedicine hub and infrastructure with interface recommendation, $11^{\text {th }}$ IEEE International Symposium on Applied Computational Intelligence and Informatics, Timisoara, Romania, 12-14 May 2016, pp. 369-374.

[16] Garai Á., Péntek I. Adaptive services with cloud architecture for telemedicine, 6th IEEE Conference on Cognitive Infocommunications, Györ, Hungary, 19-21 October 2015, pp. 369-374.

[17] Toman H., Kovacs L., Jonas A., Hajdu L, Hajdu A. Generalized weighted majority voting with an application to algorithms having spatial output, Lecture Notes in Computer Science, $7^{\text {th }}$ Hybrid Artificial Intelligent Systems Proceedings, Springer, Vol. 7209, 2012, pp. 56-67.

[18] Adamkó A., Kollár L. A system model and applications for intelligent campuses: Intelligent engineering systems, 18th International Conference on Intelligent Engineering Systems, Tihany, Hungary, 3-5 July 2014, pp. 193-198.

[19] Kota L., Jarmai K. Efficient algorithms for optimization of objects and systems. Pollack Periodica, Vol. 9, No. 1, 2014, pp. 121-132.

[20] Roland Sz. Karoly F., Márton I., Benczur A., Kollar L., Adamkó A. Framework for smart city applications based on participatory sensing, IEEE $4^{\text {th }}$ International Conference on Cognitive Infocommunications, Budapest, Hungary, 2-5 December 2013, pp. 295-300.

[21] Garai Á. Methodology for assessment validation of platform migration of robust critical ITsystems, $8^{\text {th }}$ International Conference on Applied Informatics, Eger, Hungary, 27-30 January 2010, pp. 445-448.

[22] Adamkó A., Garai Á., Péntek I. Common open telemedicine hub and interface standard recommendation, The $10^{\text {th }}$ Jubilee Conference of PhD Students on Computer Science, Szeged, Hungary, 27-29 June 2016, pp. 24-25. 
[23] Adamkó A., Arató M., Fazekas G., Juhász, I. Performance evaluation of large-scale data processing systems, Proceedings of the 7th International Conference on Applied Informatics, Eger, Hungary, 28-31 January 2007, Vol. 1, pp. 295-301.

[24] Adamkó A., Kollár L. MDA-based development of data-driven Web applications, Proceedings of the Fourth International Conference on Web Information Systems and Technologies, Funchal, Madeira, Portugal, 4-7 May 2008, Vol. 1, pp. 252-255.

[25] Neelakantan P., Reddy A. R. M. Decentralized load balancing in distributed systems. Pollack Periodica, Vol. 9, No. 2, 2014, pp. 15-28.

[26] Adenuga O. A., Kekwaletswe R. M., Coleman A. eHealth integration and interoperability issues: towards a solution through enterprise architecture, Health Information Science and Systems, Vol. 3, No. 1, 2015, pp. 1-8.

[27] Estai M., Kanagasingam Y., Xiao D., Vignarajan J., Huang B., Estie K., Tennant M. A proof-of-concept evaluation of a cloud-based store-and-forward telemedicine app for screening for oral diseases, Journal of Telemedicine and Telecare, Vol. 22, No. 6, 2016, pp. 319-325.

[28] Huang Y., Kammerdiner A. Reduction of service time variation in patient visit groups using decision tree method for an effective scheduling, International Journal of Healthcare Technology and Management, Vol. 14, No. 1-2, 2013, pp. 3-21.

[29] Kartsakli E., Antonopoulos A., Alonso L., Verikoukis C. A Cloud-assisted random linear coding medium access control protocol for healthcare applications. Sensors, Special Issue on 'Sensors Data Fusion for Healthcare', 2014, pp. 9628-9668. 